\title{
The Effects of Self-Efficacy on Cognitive Function in Patients with Acute Stroke: Verification of the Medicating Effect of Family Support
}

\author{
Sanghee Kim \\ College of Nursing, Keimyung University, Daegu, South Korea
}

Email address:

shkim07@gw.kmu.ac.kr

\section{To cite this article:}

Sanghee Kim. The Effects of Self-Efficacy on Cognitive Function in Patients with Acute Stroke: Verification of the Medicating Effect of Family Support. American Journal of Nursing Science. Vol. 10, No. 3, 2021, pp. 163-168. doi: 10.11648/j.ajns.20211003.15

Received: May 4, 2021; Accepted: May 18, 2021; Published: May 26, 2021

\begin{abstract}
Background: Post-stroke cognitive impairment (PSCI) is a common consequence of stroke. Many factors that affect cognitive function are known, but the effects of self-efficacy and family support have not been identified. Objectives: This study was a descriptive correlation investigation to determine the mediating effects of family support on the relationship between self-efficacy and cognitive function in stroke patients. Methods: This descriptive cross-sectional study recruited 339 adult patients with acute stroke. Data were collected using structured questionnaires between January and June 2020 and analyzed by multiple regression analysis using SPSS 25.0. The mediating effect of resilience on the relationship between emotional labor and job satisfaction was analyzed using multiple regression analysis according to Baron and Kenny's procedure. Results: In this study, self-efficacy in patients with acute stroke showed a positive relationship with cognitive function $(r=.467, \mathrm{p}<.05)$ and family support $(\mathrm{r}=.341, \mathrm{p}<.001)$, and family support and cognitive function also showed a positive relationship $(\mathrm{r}=.356, \mathrm{p}<.05)$. In addition, family support was shown to have a partial mediating effect on the relationship between self-efficacy and cognitive function in patients with acute stroke $(\beta=.26, \mathrm{p}<.001)$. Conclusions: To improve the cognitive function of acute stroke patients, their self-efficacy and family support must be evaluated, and interventions are necessary to enhance self-efficacy and improve family support.
\end{abstract}

Keywords: Acute Stroke, Self-Efficacy, Cognitive Function, Family Support

\section{Introduction}

A stroke occurs when the blood supply to a part of the brain is interrupted or reduced, preventing brain tissue from getting oxygen and nutrients, lasting more than 24 hours, or leading to death. There are two main causes of stroke: a blocked artery (ischemic stroke) or leaking or bursting of a blood vessel (hemorrhagic stroke) [1]. Ischemic strokes are caused by interruption of the blood supply to the brain, while hemorrhagic strokes result from the rupture of a blood vessel or an abnormal vascular structure [2]. Due to such cerebrovascular occlusion or rupture, stroke patients experience sudden consciousness changes and local neurological disorders such as body paralysis, speech impairment, muscle stiffness, dysphagia, and cognitive dysfunction for more than 24 hours [3]. In South Korea, the stroke incidence rate is 216 per 100,000 person-years, and the stroke prevalence rate is 15.9 per 1,000 persons. New stroke patients are estimated to occur every five minutes, and the stroke rate is expected to increase by about three times in 2030 [4].

Post-stroke cognitive dysfunction is primarily caused by awakening abnormalities, memory loss, attention deficit, speech loss, and reduced spatial perception. About 50-75\% of stroke patients are known to experience cognitive dysfunction, and even after three years of the diagnosis, cognitive dysfunction persists in four out of 10 people [5]. Cognitive dysfunction is an important factor affecting the recovery outcome of stroke, and it is very important to evaluate cognitive function early and provide appropriate management [6]. Recovery of brain function after stroke occurs relatively early in development, and sufficient treatment must be provided to the patient within three months, when the brain recovers most, to expect a positive 
prognosis and promote neuroplasticity, which is the key to recovery $[5,6]$.

Self-efficacy is the belief in one's capabilities to organize and execute the courses of action required to manage prospective situations. Because stroke patients require continuous self-management to recover their lost independent functions, patients' confidence that they can manage themselves well is important for continuous treatment [7]. Assistance with implementation can help change patients' health behaviors. The higher the self-efficacy of stroke patients, the higher the performance of health promotion for self-management, which is known to improve the quality of life [8]. However, many studies have been conducted of factors affecting cognitive function in stroke patients, but there has been a lack of research confirming that self-efficacy in stroke patients is an influential factor in cognitive function.

Stroke patients need help from people around them to conduct their daily lives, and, among them, family is an important support system for the long-term treatment of stroke patients [9]. It can be inferred that cognitive function is influenced not only by the patient's internal characteristics but also by external factors such as family support, as self-efficacy increases when the patient is trusted by the family or people around them.

Family support and self-efficacy have been individually identified in a small number of studies as factors affecting cognitive function in stroke patients, but neither have been verified with respect to the mediating effects of factors that can maximize patients' internal factors [9, 10]. Studies of these factors can help develop effective nursing interventions for disease treatment by identifying various applicable surrounding support factors and by strengthening the internal factors of individuals required for the long-term treatment of stroke patients [11]. In this study, we therefore aimed to provide basic data to identify the degree of self-efficacy and cognitive function of stroke patients and empirically verified the mediating effect of family support on the relationship between self-efficacy and cognitive function.

This study was conducted to explore factors such as self-efficacy and family support affecting cognitive function in patients with acute stroke. Specifically, this study aimed to identify the mediating effects of family support on self-efficacy affecting cognitive function in patients.

\section{Materials and Methods}

\subsection{Study Design and Sampling}

This study used a descriptive, cross-sectional design and was conducted from January to June 2020 in a hospital with more than 1,800 beds. This study used convenience sampling for hospitalized or outpatient clinics.

\subsection{Participants}

The participants in this study had been newly diagnosed with acute stroke and had undergone its treatment in the hospital, and they participated in the study for three months after they were discharged. The specific inclusion and exclusion criteria were as follows.

\subsubsection{Participant Inclusion Criteria}

The inclusion criteria for participants in this study were as follows: 1) patients ( $\geq 19$ years old) newly diagnosed with acute stroke by a neurologist; 2) patients who had taken the Korean Mini Mental State Examination (MMSE-K) and scored above 18-23, as evaluated by a neurologist; 3) patients who had completed the Modified Rankin Scale (MRS) and scored under 3 (i.e., the patient had moderate disability, requiring some external help but able to walk without the assistance of another individual), as evaluated by a rehabilitation specialist.

\subsubsection{Participant Exclusion Criteria}

The exclusion criteria for participants in this study were as follows: 1) patients who had been prescribed medications for hormone diseases and 2) patients who had psychological or cognitive problems that could cause communication problems before being newly diagnosed with acute stroke.

\subsection{Sample Size}

The appropriate sample size for this study was calculated using $\mathrm{G}^{*}$ Power software (G*Power version 3.1.9.2; Statistical Power Analyses Inc., L. A., USA). The multivariate regression was calculated with a power. 90 effect size as medium ( $\mathrm{f}=.15)$, a Cronbach's alpha of 0.05 , and two independent factors, and the resultant sample size was 213 subjects. Considering a dropout rate of $10 \%$, the required sample size was approximately 235 , and our total sample size was 339 , excluding insufficient questionnaires.

\subsection{Measurements}

\subsubsection{Self-efficacy}

The Stroke Self-Efficacy Questionnaire (SSEQ) was used to measure participants' self-efficacy in performing daily functional activities and self-management, such as getting in or out of bed, preparing meals, or persevering to make progress. The SSEQ is a 13-item self-report scale measuring self-efficacy judgments in specific domains of post-stroke functioning. Individuals rate their belief in their ability to achieve each of the 13 items on an 11-point Likert scale, where $0=$ not at all confident to $10=$ very confident. The scores of all items were summed to yield the total score (range 0-130). Higher scores represent higher self-efficacy in performing daily functional activities and self-management [12]. The reliability of this tool has been previously demonstrated by a Cronbach's alpha coefficient ranging from 0.85 to 0.92 , and this study showed a Cronbach's alpha of 0.89 .

\subsubsection{Cognitive Function}

The K-MMSE was used to measure participants' cognitive function. The Mini-Mental State Examination (MMSE) was first developed by Folstein in 1975 and is the most widely used screening test for cognitive function. Moreover, this tool is simple to use in clinical practice. The Korean version was 
translated and modified by Kang (1997) [13]. The K-MMSE incorporates a range of elements that include time orientation ( $0-5$ points), spatial orientation $(0-5$ points $)$, memory registration ( $0-3$ points), attention and calculation ( $0-5$ points), memory recall ( $0-3$ points), and language and space-time configuration ( $0-9$ points). The total score is 30 points, and a higher score indicates a higher degree of cognitive function. In this study, mild cognitive impairment was defined as a score between 18 and 23, and this range showed a high degree of sensitivity and specificity in a previous study. The reliability of this tool has been previously demonstrated by a Cronbach's alpha coefficient ranging from 0.80 to 0.88 , and this study showed a Cronbach's alpha of 0.81 .

\subsubsection{Family Support}

The tool for measuring family support was developed by Cobb (1976) and modified by Kang (1984) [14, 15]. This scale consists of 11 items rated on a 5-point Likert scale ( $1=$ not at all to $5=$ extremely). In the tool, the negative questions are reverse calculated, and a higher total sum score indicates a higher level of family support. The validity and reliability of this tool have been previously demonstrated by a Cronbach's alpha coefficient ranging from 0.825 to 0.913 , 15 and this study showed a Cronbach's alpha of 0.92 .

\subsection{Data Collection and Ethical Considerations}

The measurement indicators as variables in this study were collected from the participants at three months after discharge. This study was approved by the institutional review board of a single university hospital in South Korea with more than 1,800 patient beds (Reference No. C-112-13).

\subsection{Statistical Analysis}

Data were analyzed using SPSS statistical software (version 25.0; SPSS Inc., Chicago, IL, USA). The demographic and disease-related characteristics of the participants were analyzed using descriptive statistics. Pearson's correlation analysis was performed to analyze the relationships among self-efficacy, family support, and cognitive function; p-values less than.05 were considered statistically significant. The mediation analysis was conducted using the procedures described by Baron and Kenny (1986). Hierarchical regression was used to test the mediating effect of family support on the relationship between self-efficacy and cognitive function. The statistical significance of the mediating effect was analyzed using the Sobel test. All statistical results were considered statistically significant at $\mathrm{p}<0.05$.

\section{Results}

\subsection{Sociodemographic and Stroke-related Characteristics of Participants}

The sociodemographic and stroke-related characteristics of the study population are shown in Table 1 . There were more males $(n=184,54.28 \%)$ than females $(n=2,2.4 \%)$. The most common age group comprised participants in their $60 \mathrm{~s}(\mathrm{n}=161$, $47.49 \%)$, followed by those in their $50 \mathrm{~s}(\mathrm{n}=112,33.04 \%), 40 \mathrm{~s}$ $(\mathrm{n}=45,13.27 \%)$, and $30 \mathrm{~s}(\mathrm{n}=21,6.2 \%)$. The highest education level of the participants was that of high school graduate $(\mathrm{n}=153,45.14 \%)$. or $46.49 \%)$. Most participants $(\mathrm{n}=216$, $74.04 \%)$ were married, and $93.51 \%(n=317)$ lived with their families. The most common caregiver of the participants was the spouse $(n=234,69.03 \%)$. The participants in this study were relatively more religious ( $\mathrm{n}=187$ or $55.16 \%$ ) and employed $(n=211$ or $5 \%)$. Most of the patients $(n=204$, $60.18 \%$ ) earned 2,000-4000 in monthly income. The diagnosis was hemorrhagic stroke in 118 (34.81\%) patients and ischemic stroke in $221(65.19 \%)$ patients. In terms of level of disability, the mean MRS score of the participants was $2.12 \pm 1.03$, indicating premorbid disability. In terms of neurological function, the participants' mean NIHSS was $7.21 \pm 4.84$, indicating moderate stroke.

Table 1. Sociodemographic and stroke-related characteristics of participants $(N=339)$.

\begin{tabular}{|c|c|c|c|}
\hline Characteristics & Categories & n (\%) & $\mathbf{M} \pm \mathbf{S D}$ \\
\hline \multirow{2}{*}{ Gender } & Male & $184(54.28)$ & \\
\hline & Female & $155(45.72)$ & \\
\hline \multirow{5}{*}{ Age (year) } & & & $65.27 \pm 11.23$ \\
\hline & $30-39$ & $21(6.20)$ & \\
\hline & $40-49$ & $45(13.27)$ & \\
\hline & $50-59$ & $112(33.04)$ & \\
\hline & $\geq 60$ & $161(47.49)$ & \\
\hline \multirow{3}{*}{ Education level } & Middle school & 44 (12.97) & \\
\hline & High school & $153(45.14)$ & \\
\hline & $\geq$ College & $130(38.35)$ & \\
\hline \multirow{3}{*}{ Religions } & Yes & $187(55.16)$ & \\
\hline & No & $152(44.84)$ & \\
\hline & Single & $59(17.40)$ & \\
\hline Marital status & Married & $216(63.72)$ & \\
\hline Living arrangement & With family & $317(93.51)$ & \\
\hline \multirow[t]{3}{*}{ Caregiver } & Spouse & $234(69.03)$ & \\
\hline & Parent or children & $61(17.99)$ & \\
\hline & Relatives & $44(12.98)$ & \\
\hline
\end{tabular}




\begin{tabular}{llll}
\hline Characteristics & Categories & n (\%) & M \pm SD \\
\hline Monthly income (\$, USD) & $<2,000$ & $35(10.32)$ & \\
& $2,000-4,000$ & $204(60.18)$ & $100(29.50)$ \\
& $>4,000$ & $211(62.24)$ \\
Employment status & Employed & $128(37.76)$ & \\
& Unemployed & $221(65.19)$ & \\
Lesion type & Ischemic infarction & $118(34.81)$ & $2.12 \pm 1.03$ \\
Level of disability & Hemorrhagic stroke & & $7.21 \pm 4.84$ \\
Neurological function & MRS & & \\
\hline
\end{tabular}

\subsection{Descriptions of Self-efficacy, Family Support, and Cognitive Function}

The descriptions of self-efficacy, family support, and cognitive function are presented in Table 2. The mean of the participants' self-efficacy scores was $62.81 \pm 9.88$, and that of the family support scores was $2.77 \pm 0.55$. The mean cognitive function score was $21.22 \pm 1.34$, indicating mild cognitive impairment.

Table 2. Descriptions of Self-efficacy, Family Support, and Cognitive Function.

\begin{tabular}{lllll}
\hline Variables & M \pm SD & Range & Max & Min \\
\hline Self-efficacy & $62.81 \pm 9.88$ & $15 \sim 75$ & 76.6 & 35.4 \\
Family support & $2.77 \pm 0.55$ & $1 \sim 4$ & 3.59 & 1.86 \\
Cognitive function $^{\dagger}$ & $21.22 \pm 1.34$ & $0 \sim 30$ & $22.41 \pm 2.67$ & $18.68 \pm 1.56$ \\
\hline
\end{tabular}

\subsection{Relationship Among Self-efficacy, Family Support, and Cognitive Function}

correlated with family support $(\mathrm{r}=.341, \mathrm{p}<.001)$ and cognitive function $(r=.467, \mathrm{p}<.05)$. Family support was significantly correlated with cognitive function $(\mathrm{r}=.356, \mathrm{p}<.05)$.

The relationships between the independent and mediator variables are listed in Table 3. Self-efficacy was positively

Table 3. Relationship among Self-efficacy, Family Support, and Cognitive Function.

\begin{tabular}{llll}
\hline Variables & Self-efficacy & Family support & Cognitive function \\
\hline Self-efficacy & 1.00 & & 1.00 \\
Family support & $.341(<.001)$ & $.356(<.05)$ & 1.00 \\
Cognitive function $^{\dagger}$ & $.467(<.05)$ & & \\
\hline
\end{tabular}

\subsection{Mediating Effect of Family Support on the Relationship Between Self-efficacy and Cognitive Function}

Mediating effect of family support on the relationship between self-efficacy and cognitive function are presented in Table 4 and Figure 1. This study applied Baron and Kenny's methods to identify family support as having a mediating effect on the relationship between self-efficacy and cognitive function. After confirming the linear relationship between variables and confirming that there was no multicollinearity, a hierarchical regression analysis was performed. The analysis of the four steps in the process was as follows: first, self-efficacy, an independent variable, had a statistically significant correlation with cognitive function, the dependent variable $(\beta=.59, p=.021)$; second, self-efficacy had a statistically significant correlation with family support, a mediating variable $(\beta=.31, \mathrm{p}<.001)$; third, the effect of family support on cognitive function was also shown to be significant $(\beta=.30, p=.018)$, and the regression results of setting self-efficacy and family support as predictors and cognitive function as dependent variables were shown to be significant predictors of cognitive function in both self-efficacy $(\beta=.22, p=.007)$ and family support $(\beta=.22$, $\mathrm{p}<.001)$. At this step, it was confirmed that the influence on the cognitive function of self-efficacy, an independent variable, decreased compared to step 1. According to Baron and Kenny (1986), if the independent variable influence in the final step decreases from the first step and continues to be significant, there is a partial mediating effect. To verify the statistical significance of the mediating effect of family support, Sobel tests were conducted, and family support had a significant partial mediating effect on the effect of self-efficacy on cognitive function $(Z=4.67, \mathrm{p}<.001)$.

Table 4. Mediating Effect of Family Support on the Relationship between Self-efficacy and Cognitive Function.

\begin{tabular}{|c|c|c|c|c|c|c|}
\hline Step & Path & B & SE & $\beta$ & $\mathbf{R}^{2}$ & $p$ \\
\hline Step1 & Self-efficacy $\rightarrow$ Cognitive function & 0.66 & 0.14 & .59 & 28 & .021 \\
\hline Step2 & Self-efficacy $\rightarrow$ Family support & 0.31 & 0.15 & .31 & 31 & $<.001$ \\
\hline Step3 & Family support $\rightarrow$ Cognitive function & 0.29 & 0.01 & .30 & 38 & .018 \\
\hline Step4 & $\begin{array}{l}\text { Self-efficacy } \\
\text { Family support }\end{array}$ & $\begin{array}{l}0.21 \\
0.22\end{array}$ & $\begin{array}{l}0.15 \\
0.06\end{array}$ & $\begin{array}{l}.22 \\
.26\end{array}$ & .22 & $\begin{array}{l}.007 \\
<.001\end{array}$ \\
\hline
\end{tabular}




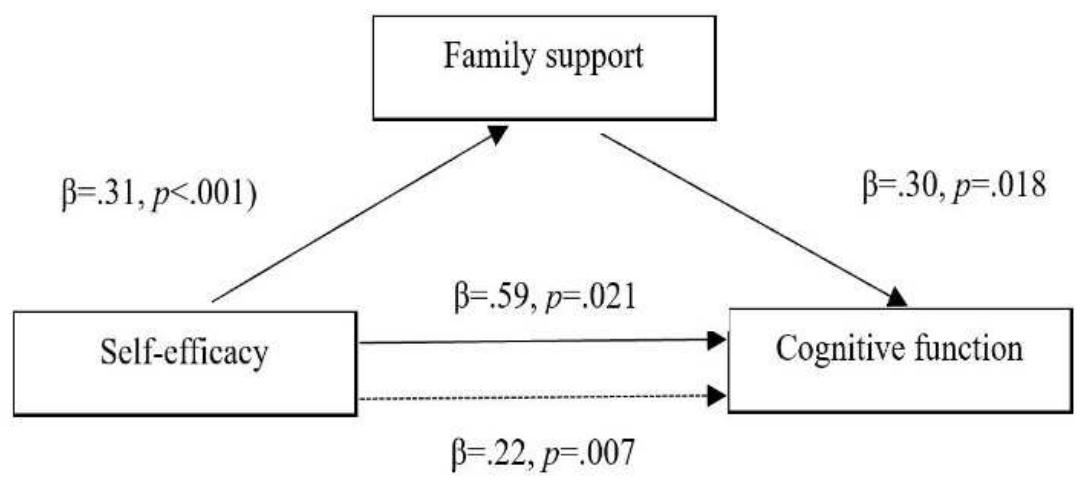

Figure 1. Mediating effect of family support in the relationship between self-efficacy and cognitive function.

\section{Discussion}

It is estimated that approximately $50-75 \%$ of patients with acute stroke experience cognitive impairment, and patients with cognitive impairment often have difficulty performing their daily activities [5]. Cognitive dysfunction is an important predictor of the recovery of function in stroke patients, and cognitive dysfunction is likely to recover if there are appropriate interventions, as brain function recovers the most within three months, during the relatively early stages of the onset [16]. Therefore, early detection of and intervention in factors affecting cognitive function in stroke patients can effectively manage the disease. Stroke is a disease that requires long-term care to manage and prevent the recurrence of after-effect disorders, including cognitive impairment [2, 5]. Voluntary and continuous patient efforts to adopt health promotion activities and lifestyles are very important. Self-efficacy is an important factor for improving stroke patients' quality of life, continuous treatment, and implementation. Previous studies have shown its relation to an increase in patients' self-care and health promotion performance [17]. In this study, the correlation between self-efficacy and cognitive function in patients with acute stroke was significantly high. Self-efficacy motivates the acceptance of altered functional changes, increases medication adherence, and actively participates in rehabilitation in stroke patients. Self-efficacy has also been observed to be an important factor in restoring cognitive function in stroke patients, which is ultimately important in health-promoting activities for cognitive recovery.

Types of social support are classified as family, friends, and communities. These are important support systems for diseases that require long-term treatment and care, such as stroke. This support reduces emotional distress in stroke patients and allows them to positively embrace trauma experiences and implications $[10,15,18]$. For stroke patients with limited performance in their daily lives, families, as an important resource that most directly provides help after discharge, stimulate patients' rehabilitation motivation for disease relief and recovery [19, 20]. In this study, family support and cognitive function in acute stroke patients were significantly correlated, which can be interpreted to mean that family support can enhance the recovery of a patient from both physical dysfunction and cognitive dysfunction based on the belief and trust gained from those around him. Therefore, the development of an integrated and multidimensional intervention program for cognitive recovery in acute stroke patients will need to include the role of the family, which is an external factor, in addition to the patient's internal characteristics. In this study, family support in patients with acute stroke had a partial mediating effect on the effect of self-efficacy on cognitive function. This result means that even if there is not a direct intervention using only the patient's self-efficacy, their recovery of cognitive function can be assisted by family support. Several prior studies have shown that family support helps in the practice of health behaviors by reducing uncertainty about the disease and strengthening rehabilitation motivation in patients with acute stroke. Therefore, to provide a multidirectional approach to restoring cognitive function in patients with acute stroke, it is necessary to develop interventions related to family support that can increase self-efficacy and the internal will of patients. The specificity of this study is that it verified the relationship between self-efficacy and cognitive function in patients with acute stroke, which has not been well addressed in existing studies, and derived the influence of family support from these relationships. It is also meaningful in that it presents practical implications for family support utilization as a variety of factors to help patients with acute stroke recover cognitive function.

\section{Conclusions}

This study confirmed the partially mediated effect of family support on the relationship between self-efficacy and cognitive function in patients with acute stroke. Therefore, self-efficacy, an internal characteristic of the patient, should be identified to restore cognitive function in patients with acute stroke, Moreover, applying mediation that can increase family support by the primary caregiver could help patients with acute stroke.

\section{Findings}

This research was supported by the Dongsan Hospital Grant of Keimyung University in 2019. 


\section{References}

[1] Kuriakose D, Xiao Z. Pathophysiology and Treatment of Stroke: Present Status and Future Perspectives. Int J Mol Sci. 2020; 21 (20): 7609. doi: 10.3390/ijms21207609.

[2] Chugh C. Acute Ischemic Stroke: Management Approach. Indian J Crit Care Med. 2019; 23 (2): S140-S146. doi: 10.5005/jp-journals-10071-23192.

[3] Ojaghihaghighi S, Vahdati SS, Mikaeilpour A, Ramouz A. Comparison of neurological clinical manifestation in patients with hemorrhagic and ischemic stroke. World J Emerg Med. 2017; 8 (1): 34-38. 10.5847/wjem.j.1920-8642.2017.01.006.

[4] Kim JY, Kang K, Kang J, Koo J, Kim DH, Kim BJ, et al. Executive Summary of Stroke Statistics in Korea 2018: A Report from the Epidemiology Research Council of the Korean Stroke Society. J Stroke. 2019 Jan; 21 (1): 42-59. doi: 10.5853/jos.2018.03125.

[5] Sun JH, Tan L, Yu JT. Post-stroke cognitive impairment: epidemiology, mechanisms and management. Ann Transl Med. 2014; 2 (8): 80. doi: 10.3978/j.issn.2305-5839.2014.08.05.

[6] Obaid M, Flach C, Marshall I, D A Wolfe C, Douiri A. Long-Term Outcomes in Stroke Patients with Cognitive Impairment: A Population-Based Study. Geriatrics (Basel). 2020; 5 (2): 32. doi: 10.3390/geriatrics5020032.

[7] Szczepańska-Gieracha J, Mazurek J. The Role of Self-Efficacy in the Recovery Process of Stroke Survivors. Psychol Res Behav Manag. 2020; 13: 897-906. doi: 10.2147/PRBM.S273009.

[8] Dallolio L, Messina R, Calugi S, Fugazzaro S, Bardelli R, Rucci P, Fantini MP, Cavalli E, Taricco M. Self-management and self-efficacy in stroke survivors: validation of the Italian version of the Stroke Self-Efficacy Questionnaire. Eur J Phys Rehabil Med. 2018; 54 (1): 68-74. doi: 10.23736/S1973-9087.16.04451-8.

[9] Tsai PC, Yip PK, Tai JJ, Lou MF. Needs of family caregivers of stroke patients: a longitudinal study of caregivers' perspectives. Patient Prefer Adherence. 2015; 9: 449-457. doi: 10.2147/PPA.S77713.

[10] Creasy KR, Lutz BJ, Young ME, Stacciarini JM. Clinical Implications of Family-Centered Care in Stroke Rehabilitation. Rehabil Nurs. 2015; 40 (6): 349-359. doi: 10.1002/rnj.188.
[11] Creasy KR, Lutz BJ, Young ME, Stacciarini JM. Change of cognitive functions after stroke with rehabilitation systems. Transl Neurosci. 2019; 10: 118-124. doi: 10.1515/tnsci-2019-0020.

[12] Ma HI, Hung PH, Lin SH, Chuang IC, Wu CY. The role of self-efficacy in the predictive relationship of motor ability to functional performance after task-related training in stroke: A secondary analysis of longitudinal data. Arch Phys Med Rehabil. 2021; S0003-9993 (21) 00295-1. doi: 10.1016/j.apmr.2021.03.017.

[13] Kim BR, Chun MH, Kim LS, Park JY. Effect of Virtual Reality on Cognition in Stroke Patients. Ann Rehabil Med. 2011; 3 (4): 450-459. doi: 10.5535/arm.2011.35.4.450.

[14] Cobb S. Presidential Address-1976. Social support as a moderator of life stress. Psychosom Med 1976; 38: 300-14. doi: 10.1097/00006842-197609000-00003.

[15] Han J, Lee HI, Shin YI, Son JH, Kim SY, Kim DY. Factors influencing return to work after stroke: the Korean Stroke Cohort for Functioning and Rehabilitation (KOSCO) Study. BMJ Open. 2019; 9 (7): e028673. doi: 10.1136/bmjopen-2018-028673.

[16] Al-Qazzaz NK, Ali SH, Ahmad SA, Islam S, Mohamad K. Cognitive impairment and memory dysfunction after a stroke diagnosis: a post-stroke memory assessment. Neuropsychiatr Dis Treat. 2014; 10: 1677-1691. doi: 10.2147/NDT.S67184.

[17] Stewart JC, Lewthwaite R, Rocktashel J, Winstein CJ. Self-efficacy and Reach Performance in Individuals with Mild Motor Impairment Due to Stroke. Neurorehabil Neural Repair. 2019; 33 (4): 319-328. doi: 10.1177/1545968319836231.

[18] Lutz BJ, Young ME, Creasy KR, Martz C, Eisenbrandt L, Brunny JN, et al. Improving Stroke Caregiver Readiness for Transition From Inpatient Rehabilitation to Home. $\begin{array}{lllll}\text { Gerontologist. 2017; } 57 \quad \text { (5): } 880-889 . & \text { doi: }\end{array}$ 10.1093/geront/gnw135.

[19] Lo Coco D, Lopez G, Corrao S. Cognitive impairment and stroke in elderly patients. Vasc Health Risk Manag. 2016; 12: 105-116. doi: 10.2147/VHRM.S75306.

[20] Tang EYH, Price C, Stephan BCM, Robinson L, Exley C. Impact of Memory Problems Post-stroke on Patients and Their Family Carers: A Qualitative Study. Front Med (Lausanne). 2020; 7: 267. doi: 10.3389/fmed.2020.00267. 